\title{
Association of the XPA A23G polymorphism with the risk of head and neck carcinomas: Evidence from 5,491 subjects
}

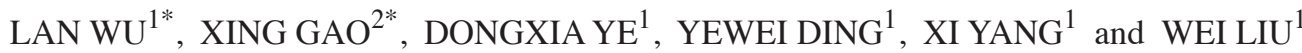 \\ ${ }^{1}$ Shanghai Key Laboratory of Stomatology, Ninth People's Hospital, Shanghai Jiao Tong \\ University School of Medicine, Shanghai 200001; ${ }^{2}$ Department of Oral and Maxillofacial Surgery, \\ XiangYa Hospital, Central South University, Changsha, Hunan 410011, P.R. China
}

Received December 17, 2014; Accepted February 16, 2015

DOI: $10.3892 / \mathrm{mco} .2015 .513$

\begin{abstract}
The $X P A$ gene participates in modulating DNA damage recognition during the DNA nucleotide excision repair process. Current data regarding the association of the $X P A$ A23G polymorphism with the risk of head and neck squamous cell carcinoma (HNSCC) remain controversial, and meta-analyses focusing on the HNSCC risk and this polymorphism are limited. Therefore, the aim of the present study was to derive a more precise estimation of this association by a meta-analysis of all the eligible studies. Odds ratios (ORs) with $95 \%$ confidence intervals (CI) were assessed for the strength of the associations in eight studies, including 5,491 subjects (2,409 HNSCC cases and 3,082 controls). The overall analysis revealed that the XPA A23G polymorphism was not significantly associated with the overall HNSCC risk. Consistently, there was no evidence for the association between the XPA A23G polymorphism and HNSCC risk in subgroup analyses based on ethnicity and the source of controls. However, the significant associations in oral carcinoma with the increased risk among the XPA heterozygote (AG vs. AA: OR, 1.58; 95\% CI, 1.06-2.37; $\mathrm{P}_{\text {heterogeneity }}=0.23$, $\mathrm{I}^{2}=30 \%$ ) and dominant (AG $+\mathrm{GG}$ vs. AA: OR, $1.53 ; 95 \% \mathrm{CI}$, 1.04-2.23; $\mathrm{P}_{\text {heterogeneity }}=0.21, \mathrm{I}^{2}=36 \%$ ) models were observed in the subgroup analysis by tumor site. In conclusion, the meta-analysis suggested that the XPA A23G polymorphism was not associated with overall HNSCC susceptibility, but it was associated with oral carcinoma susceptibility and it may be a risk factor for oral carcinoma. Further well-designed and large studies are required to confirm these associations.
\end{abstract}

Correspondence to: Dr Xi Yang or Dr Wei Liu, Shanghai Key Laboratory of Stomatology, Ninth People's Hospital, Shanghai Jiao Tong University School of Medicine, 639 Zhizaoju Road, Shanghai 200001, P.R. China

E-mail: yangxi16@163.com

E-mail: liuweb@hotmail.com

*Contributed equally

Key words: XPA A23G, polymorphism, head and neck, oral cancer, meta-analysis

\section{Introduction}

Head and neck squamous cell carcinoma (HNSCC), including the oral cavity, pharynx and larynx, constitutes the sixth most common malignancy worldwide, and the pathogenesis remains unclear (1). Although increasing evidence suggests that environmental factors and chemical carcinogens, such as tobacco use and excessive alcohol consumption, are possible etiological factors contributing to HNSCC susceptibility, only a small fraction of people exposed to these carcinogens will develop HNSCC $(2,3)$. HNSCC development is widely recognized as a stepwise process with the involvement of a series of genetic alterations, and host genetic factors may also be involved in the carcinogenesis (4). The identification of a predictive model of risk polymorphisms may aid in early diagnosis and in understanding disease progression in a subset of cancer patients.

The xeroderma pigmentosum group $\mathrm{A}(X P A)$ gene codes for a zinc-finger DNA-binding protein, and it is a part of the nucleotide excision repair (NER) complex, which is responsible for repair of ultraviolet radiation-induced photoproducts and DNA adducts by chemical carcinogens $(5,6)$. Additionally, $X P A$ is involved in a step of damage recognition, and plays a key role in global genome and transcription-coupled repair pathways (7). A common single-nucleotide polymorphism of XPA A23G, rs1800975, is an A-to-G point mutation in the 5 ' noncoding region of four nucleotides upstream of the start codon of XPA, which has been identified and extensively researched (8-10).

Thus far, several molecular epidemiological studies have investigated the association between the XPA A23G polymorphism and HNSCC risk (11-18). However, the results remain inconsistent and controversial, partially due to the relatively small sample size of independent studies and sampling effects. Meta-analysis allow stronger conclusions for identifying certain models of risk markers, which may aid in screening, early diagnosis and/or therapy in the clinic $(19,20)$. To the best of our knowledge, no previous meta-analysis has focused on the XPA A23G polymorphism and HNSCC risk. Therefore, a meta-analysis was performed on all the eligible studies to achieve a more precise estimation of this association, as well as to investigate the source of heterogeneity and potential bias. 


\section{Materials and methods}

Literature search strategy. The electronic databases PubMed, Medline, Embase and China National Knowledge Infrastructure were searched for relevant studies to include in the meta-analysis, without restriction on language and publication year (until Nov 20, 2014). The following search terms were used: ('Xeroderma pigmentosum group A' OR 'XPA' OR 'rs1800975') AND ('carcinoma' OR 'cancer' OR 'malignancy' OR 'neoplasm' OR 'tumour' OR 'tumor') AND ('head and neck' or 'oral' or 'oropharyngeal' or 'nasopharyngeal' or 'laryngopharyngeal' or 'hypopharyngeal' or 'laryngeal'). The potential associated studies were reviewed to evaluate their suitability for inclusion in the meta-analysis. Additional relevant studies were identified through the references cited in the retrieved studies or reviews on this topic.

Inclusion and exclusion criteria. The inclusion criteria for the eligible studies were as follows: i) Human case-control studies; ii) evaluation of the XPA A23G (rs1800975) polymorphism and HNSCC risk; iii) sufficient genotype data to estimate an odds ratio (OR) and 95\% confidence interval (95\% CI); and iv) histologically confirmed diagnosis of HNSCC. Accordingly, the exclusion criteria were as follows: i) Not a case-control study; ii) duplicate or overlapping studies; and iii) no usable data reported.

Data extraction. According to the selection criteria, all the relevant crude data were extracted from each eligible study independently by two investigators. Inconsistencies were discussed until a consensus was obtained. The following items were extracted from each study: First author name, year of publication, country of origin, tumor site, ethnicity, source of control (population- or hospital-based controls), genotyping methods, number of cases and controls, characteristics of cancer cases and controls, genotype frequencies for cases and controls.

Statistic analysis. Hardy-Weinberg equilibrium (HWE) of the control group of each study was measured using the Pearson's goodness-of-fit $\chi^{2}$ test. The strength of the association between the XPA A23G polymorphism and HNSCC and OSCC risk was determined by OR with $95 \%$ CI. In the overall and subgroup meta-analysis, pooled ORs and 95\% CIs for the heterozygote (AG vs. AA), homozygote (GG vs. AA), dominant (AG+GG vs. AA) and recessive models (GG vs. AG+AA) were calculated. The allele comparison ( $G$ vs. A) was conducted as an additive model. The statistical significance of the pooled OR was evaluated using the Z-test, and the heterogeneity of the ORs was tested by the $\chi^{2}$-based Q-test and $\mathrm{I}^{2}$ statistics. When the result of the heterogeneity test showed $\mathrm{P}>0.1$, ORs were pooled according to the fixed-effects model (Mantel-Haenszel model). Otherwise, the random-effects model (DerSimonian and Laird model) was selected. Additionally, the Egger's test and Begg's funnel plot were used to measure the potential publication bias. All the statistical analyses were performed with the STATA 12.0 software (Stata Corporation, College Station, TX, USA) and Review Manager 5.0 (The Cochrane Collaboration, Oxford, UK), using two-tail P-values.

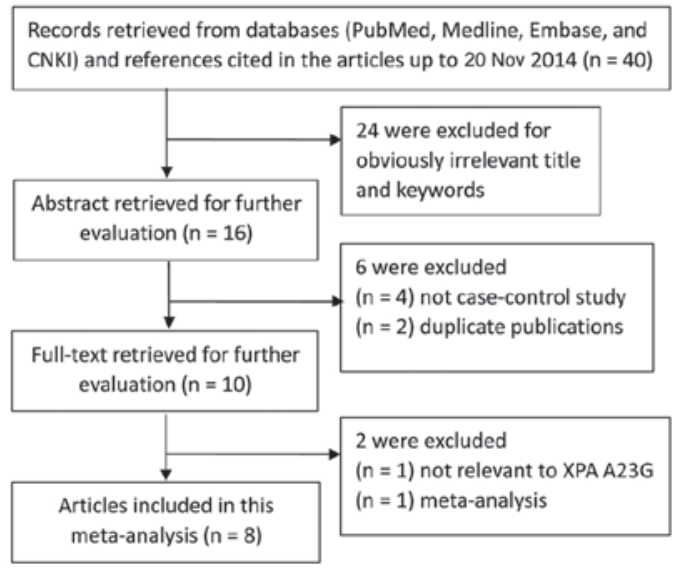

Figure 1. Flow diagram of the study selection process.

\section{Results}

Study characteristics. Of the 40 potentially relevant studies, 30 were excluded and 10 full-text studies were retrieved for detailed evaluation from the search of the published literature. During the extraction of data, one study by Schena et al (18) that was not relevant to the XPA A23G polymorphism was excluded; and one meta-analysis by Liu et al (8) was excluded. Therefore, eight eligible studies, including 2,409 HNSCC cases and 3,082 controls, were identified in the final meta-analysis (Fig. 1). Characteristics of the eligible studies are summarized in Table I. All the studies were case-control studies, including two oral carcinoma studies, three laryngeal carcinoma studies, and three mixed head and neck carcinomas studies. Four of the eight studies were conducted in the Asian population and four were conducted in Caucasians. There were five hospital-based and three population-based control studies. A polymerase chain reaction-restriction fragment length polymorphism (PCR-RFLP) assay was performed in four of these studies. The detailed variant genotype distributions of the XPA A23G polymorphism for the HNSCC cases and controls in the individual studies are listed in Table I.

Meta-analysis results. The associations of the XPA A23G polymorphism with the HNSCC risk are summarized in Table II. In the overall analysis, no significant association was observed in any genetic model; heterozygote (AG vs. AA: OR, 1.06; 95\% CI, 0.90-1.24), homozygote (GG vs. AA: OR, 1.06; 95\% CI, 0.90-1.25), dominant (AG+GG vs. AA: OR, 1.11; 95\% CI, 0.95-1.29), recessive models (GG vs. AG+AA: OR, 1.05; 95\% CI, 0.94-1.18), and allele comparison (G vs. A: OR, 0.94; 95\% CI, 0.75-1.18). Consistently, there was no evidence for the association between the XPA A23G polymorphism and HNSCC risk in the subgroup analyses based on ethnicity and source of controls in all the genetic models (Table II). In the stratified analysis by tumor site, there was also no evidence for the association between the XPA A23G polymorphism and laryngeal carcinoma and mixed HNSCC risk in any genetic model. However, significant associations in oral carcinoma with increased risk among the $X P A$ heterozygote (AG vs. AA: OR, 1.58; 95\% CI, 1.06-2.37; $\mathrm{P}_{\text {heterogeneity }}=0.23, \mathrm{I}^{2}=30 \%$ ) and 
Table I. Study characteristics in the meta-analysis.

\begin{tabular}{|c|c|c|c|c|c|c|c|c|c|c|c|c|c|c|}
\hline \multirow{2}{*}{$\begin{array}{l}\text { First author, } \\
\text { year (ref.) }\end{array}$} & \multirow[b]{2}{*}{ Country } & \multirow[b]{2}{*}{ Ethnicity } & \multirow[b]{2}{*}{ Tumor site } & \multirow{2}{*}{$\begin{array}{c}\text { Control } \\
\text { source }\end{array}$} & \multirow[b]{2}{*}{ Genotyping } & \multirow{2}{*}{$\begin{array}{l}\text { HWE } \\
\text { control }\end{array}$} & \multicolumn{2}{|c|}{ Sample, $\mathrm{n}$} & \multicolumn{3}{|c|}{ Case } & \multicolumn{3}{|c|}{ Control } \\
\hline & & & & & & & Case & Control & $\mathrm{AA}$ & $\mathrm{AG}$ & GG & $\mathrm{AA}$ & AG & GG \\
\hline $\begin{array}{l}\text { Sugimura, } \\
2006 \text { (11) }\end{array}$ & Japan & Asian & Oral & Hospital & PCR-RFLP & 0.05 & 122 & 241 & 23 & 65 & 34 & 74 & 105 & 62 \\
\hline $\begin{array}{l}\text { Hall, } \\
2006 \text { (12) }\end{array}$ & CEEC & Caucasian & Mixed & Hospital & TaqMan & 0.22 & 597 & 770 & 75 & 247 & 275 & 98 & 375 & 297 \\
\hline $\begin{array}{l}\text { An, } \\
2007 \text { (13) }\end{array}$ & USA & Caucasian & Mixed & Hospital & TaqMan & $<0.05$ & 829 & 854 & 110 & 360 & 359 & 128 & 346 & 380 \\
\hline $\begin{array}{l}\text { Bau, } \\
2007 \text { (14) }\end{array}$ & China & Asian & Oral & Population & PCR-RFLP & 0.90 & 154 & 105 & 38 & 84 & 32 & 29 & 53 & 23 \\
\hline $\begin{array}{l}\text { Abbasi, } \\
2009(15)\end{array}$ & Germany & Caucasian & Laryngeal & Population & PCR-RFLP & 0.72 & 246 & 644 & 30 & 109 & 107 & 72 & 281 & 291 \\
\hline $\begin{array}{l}\text { Jelonek, } \\
2010(16)\end{array}$ & Poland & Caucasian & Mixed & Population & PCR-RFLP & 0.06 & 75 & 82 & 12 & 29 & 34 & 7 & 47 & 28 \\
\hline $\begin{array}{l}\text { Lu, } \\
2014 \text { (17) }\end{array}$ & China & Asian & Laryngeal & Hospital & MALDI-TOF & $<0.05$ & 176 & 176 & 43 & 55 & 78 & 40 & 54 & 82 \\
\hline $\begin{array}{l}\mathrm{Li}, \\
2014 \text { (18) }\end{array}$ & China & Asian & Laryngeal & Hospital & MALDI-TOF & $<0.05$ & 210 & 210 & 52 & 67 & 91 & 47 & 63 & 100 \\
\hline
\end{tabular}

HWE, hardy-weinberg equilibrium; PCR-RFLP, polymerase chain reaction and restriction fragment length polymorphism; CEEC, Central and Eastern European Countries MALDI-TOF, matrix-assisted laser desorption/ionization time-of-flight.

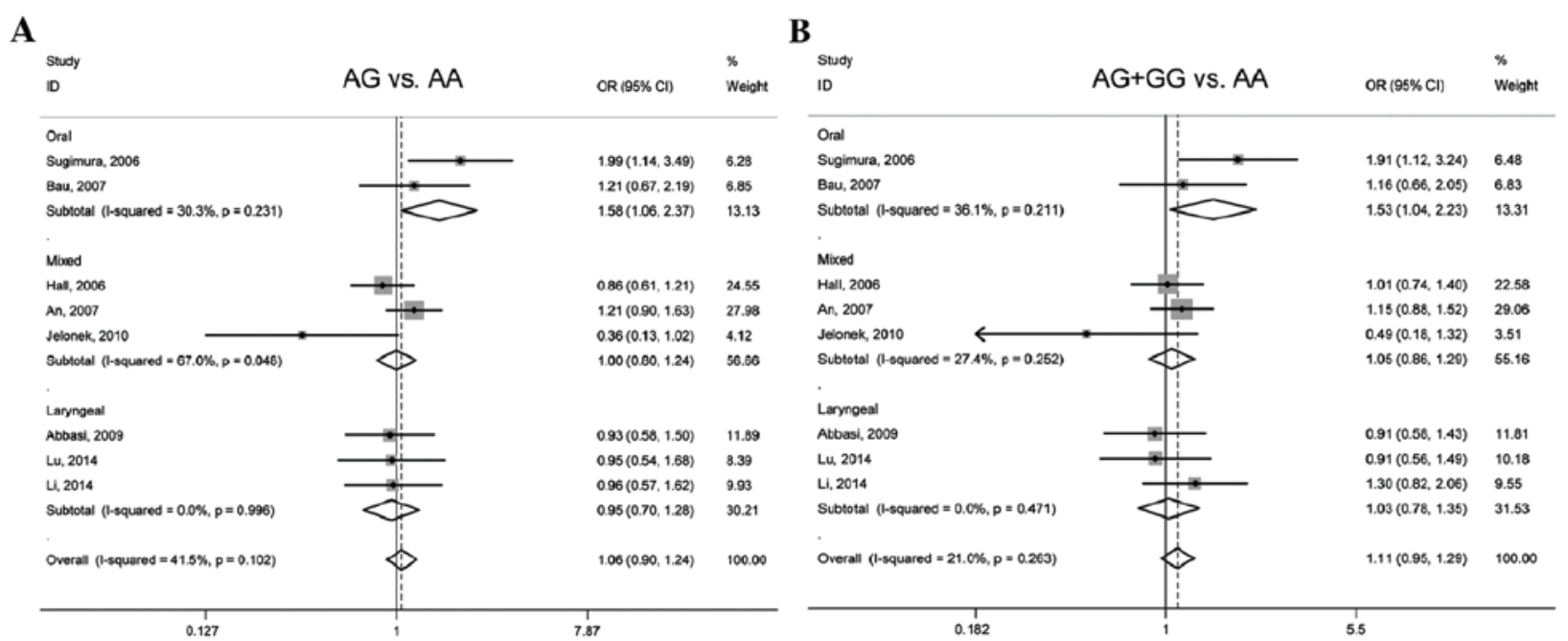

Figure 2. Forest plots of the meta-analysis of the XPA A23G polymorphism and head and neck squamous cell carcinoma stratified by oral, laryngeal and mixed head and neck cancers. (A) Heterozygote (AG vs. AA) and (B) dominant (AG+GG vs. AA) genetic models.

dominant (AG+GG vs. AA: OR, 1.53; 95\% CI, 1.04-2.23; $\mathrm{P}_{\text {heterogeneity }}=0.21, \mathrm{I}^{2}=36 \%$ ) models were observed. Forest plots for meta-analysis of these two significant genetic models are shown in Fig. 2.

Test of heterogeneity. As presented in Table II, there was no evidence of heterogeneity in the vast majority of the overall and subgroup analyses among all the genetic models $(\mathrm{P}>0.1$ for heterogeneity, Q test), and these ORs were analyzed with the fixed-effects model. Heterogeneity was found in only a few of the analyses $(\mathrm{P}<0.1$ for heterogeneity, $\mathrm{Q}$ test $)$, and these ORs were analyzed with the random-effects model.
Publication bias. Publication bias of literature was examined using the Egger's test and Begg's test. As presented in Table III, the Egger's test indicated no evidence for publication bias for all the genetic models (all $\mathrm{P}>0.05$ ), which was confirmed by Begg test (all $\mathrm{P}>0.05$ ). Fig. 3 showed that the shape of the Begg's funnel plots did not reveal any evidence of clear asymmetry in the allele G vs. A model.

\section{Discussion}

The XPA protein is involved in the DNA damage recognition process and plays a pivotal role in the NER pathway, which 
Table II. Summary of pooled odds ratios (ORs) with $95 \%$ confidence interval (CI) in the meta-analysis.

\begin{tabular}{|c|c|c|c|c|c|c|c|c|}
\hline \multirow{2}{*}{$\begin{array}{l}\text { Group } \\
\text { (No. of case/control) }\end{array}$} & \multirow[b]{2}{*}{ Genotype } & \multicolumn{4}{|c|}{ Test of association } & \multicolumn{3}{|c|}{ Test of heterogeneity } \\
\hline & & OR $(95 \% \mathrm{CI})$ & $\mathrm{Z}$ & P-value & Model & $\chi^{2}$ & P-value & $\mathrm{I}^{2}, \%$ \\
\hline \multirow{5}{*}{$\begin{array}{l}\text { Overall } \\
(2409 / 3082)\end{array}$} & AG vs. AA & $1.06(0.90-1.24)$ & 0.69 & 0.49 & Fixed & 11.97 & 0.10 & 41 \\
\hline & GG vs. AA & $1.06(0.90-1.25)$ & 0.70 & 0.48 & Fixed & 5.77 & 0.57 & 0 \\
\hline & $\mathrm{AG}+\mathrm{GG}$ vs. AA & $1.11(0.95-1.29)$ & 1.23 & 0.22 & Fixed & 8.86 & 0.26 & 21 \\
\hline & GG vs. AG+AA & $1.05(0.94-1.18)$ & 0.91 & 0.36 & Fixed & 10.60 & 0.16 & 34 \\
\hline & G vs. A & $0.94(0.75-1.18)$ & 0.52 & 0.61 & Random & 47.73 & $<0.05$ & 85 \\
\hline \multicolumn{9}{|l|}{ Ethnicity } \\
\hline \multirow{5}{*}{$\begin{array}{l}\text { Asian } \\
(662 / 732)\end{array}$} & AG vs. AA & $1.22(0.92-1.61)$ & 1.39 & 0.16 & Fixed & 4.48 & 0.21 & 33 \\
\hline & GG vs. AA & $1.03(0.77-1.36)$ & 0.18 & 0.86 & Fixed & 3.97 & 0.26 & 24 \\
\hline & $\mathrm{AG}+\mathrm{GG}$ vs. AA & $1.56(0.92-2.63)$ & 1.66 & 0.10 & Random & 13.32 & $<0.05$ & 77 \\
\hline & GG vs. AG+AA & $0.93(0.74-1.17)$ & 0.63 & 0.53 & Fixed & 0.80 & 0.85 & 0 \\
\hline & G vs. A & $0.84(0.50-1.44)$ & 0.62 & 0.53 & Random & 35.43 & $<0.05$ & 92 \\
\hline \multirow{5}{*}{$\begin{array}{l}\text { Caucasion } \\
(1747 / 2350)\end{array}$} & AG vs. AA & $0.99(0.81-1.20)$ & 0.14 & 0.89 & Fixed & 6.14 & 0.11 & 51 \\
\hline & GG vs. AA & $1.03(0.81-1.30)$ & 0.22 & 0.83 & Fixed & 0.99 & 0.80 & 0 \\
\hline & $\mathrm{AG}+\mathrm{GG}$ vs. AA & $1.03(0.85-1.24)$ & 0.29 & 0.77 & Fixed & 3.12 & 0.37 & 4 \\
\hline & GG vs. AG+AA & $1.12(0.88-1.41)$ & 0.92 & 0.36 & Random & 8.29 & 0.04 & 64 \\
\hline & G vs. A & $1.06(0.96-1.16)$ & 1.17 & 0.24 & Fixed & 3.39 & 0.35 & 11 \\
\hline \multicolumn{9}{|l|}{ Control source } \\
\hline \multirow{5}{*}{$\begin{array}{l}\text { Hospital } \\
(1934 / 2251)\end{array}$} & AG vs. AA & $1.10(0.92-1.32)$ & 1.05 & 0.29 & Fixed & 7.22 & 0.12 & 45 \\
\hline & GG vs. AA & $1.10(0.92-1.32)$ & 1.04 & 0.30 & Fixed & 4.49 & 0.34 & 11 \\
\hline & $\mathrm{AG}+\mathrm{GG}$ vs. AA & $1.39(0.95-2.05)$ & 1.69 & 0.09 & Random & 19.76 & $<0.05$ & 80 \\
\hline & GG vs. AG+AA & $1.04(0.86-1.27)$ & 0.42 & 0.67 & Random & 8.18 & 0.09 & 51 \\
\hline & G vs. A & $0.91(0.65-1.26)$ & 0.58 & 0.56 & Random & 47.31 & $<0.05$ & 92 \\
\hline \multirow{5}{*}{$\begin{array}{l}\text { Population } \\
(475 / 831)\end{array}$} & AG vs. AA & $0.91(0.64-1.29)$ & 0.52 & 0.60 & Fixed & 3.94 & 0.14 & 49 \\
\hline & GG vs. AA & $0.90(0.62-1.31)$ & 0.54 & 0.59 & Fixed & 0.40 & 0.82 & 0 \\
\hline & $\mathrm{AG}+\mathrm{GG}$ vs. AA & $0.92(0.66-1.28)$ & 0.49 & 0.62 & Fixed & 2.23 & 0.33 & 10 \\
\hline & GG vs. $A G+A A$ & $1.01(0.79-1.29)$ & 0.08 & 0.93 & Fixed & 2.29 & 0.32 & 12 \\
\hline & G vs. A & $0.98(0.83-1.17)$ & 0.20 & 0.84 & Fixed & 0.41 & 0.81 & 0 \\
\hline
\end{tabular}

Table III. Publication bias test for the XPA A23G polymorphism.

\begin{tabular}{|c|c|c|c|c|}
\hline \multirow[b]{2}{*}{ Genotype } & \multicolumn{2}{|c|}{ Egger's test } & \multicolumn{2}{|c|}{ Begg's test } \\
\hline & Coefficient & $95 \% \mathrm{CI}$ & P-value & P-value \\
\hline AG vs. AA & -1.012 & $-4.602-2.578$ & 0.516 & 0.902 \\
\hline GG vs. AA & -0.595 & $-2.972-1.783$ & 0.563 & 0.902 \\
\hline $\mathrm{AG}+\mathrm{GG}$ vs. $\mathrm{AA}$ & -0.670 & $-3.871-2.492$ & 0.615 & 0.711 \\
\hline GG vs. $A G+A A$ & 0.151 & $-2.554-2.857$ & 0.931 & 0.386 \\
\hline G vs. A & -0.293 & $-3.019-2.432$ & 0.801 & 0.711 \\
\hline
\end{tabular}

CI, confidence interval.

is a key defense mechanism against the major carcinogens of HNSCC (21-23). The A23G polymorphism regulated XPA gene expression through transcriptional and post-transcriptional control mechanisms. XPA A23G genetic variations thus have the potential to influence protein function and subsequently DNA

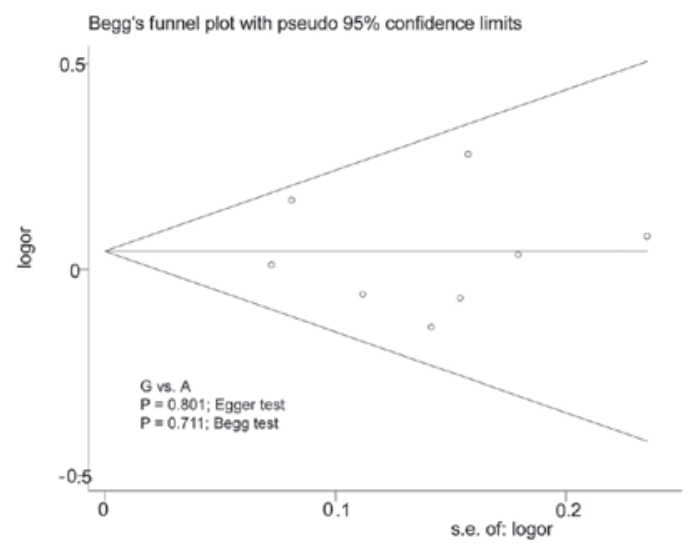

Figure 3. Begg's funnel plots and Egger's test. s.e. standard error; or, odds ratio.

repair capacity (8-10). Several studies on the association of the $X P A$ A23G polymorphism with HNSCC susceptibility have been published (11-18). However, the results are contradictory. The present study aimed to obtain a more accurate estimation 
of the association between the XPA A23G polymorphism and HNSCC susceptibility by performing a meta-analysis from all the eligible case-control studies published so far.

A previous meta-analysis conducted by Liu et al (8) of the $X P A$ A23G polymorphism and the risk of all the different types of cancer, including HNSCC, observed a significant association of an increased risk of HNSCC with the recessive model (GG vs. AG+AA: OR, 1.19; 95\% CI, 1.02-1.38). The previous meta-analysis included five studies of HNSCC $(1,194$ cases and 1,892 controls), and the present study extended the data, including eight independent studies of HNSCC $(2,409$ cases and 3,082 controls). Additionally, the present study focused on the association between the XPA A23G polymorphism and the risk of HNSCC. However, the results of the overall analysis showed that the XPA A23G polymorphism in any genetic model is not associated with the HNSCC risk when all the eligible studies were pooled together, suggesting that this polymorphism may not play a key role in HNSCC susceptibility.

A subgroup meta-analysis was performed of the XPA A23G polymorphism according to ethnicity, control source and tumor site. In the subgroup analyses by ethnicity and control source, no significant associations were identified in any genetic model among the HNSCC studies, in conformity with the results of the overall analysis (Table II). However, an increased risk of oral carcinoma was observed among the XPA A23G heterozygote and dominant carriers in the subgroup analysis by tumor site, and no significant associations were found in the laryngeal carcinoma and mixed HNSCC (Fig. 2). This data suggested that there may be a tissue-specific cancer susceptibility for the XPA A23G polymorphism and the different roles of the same polymorphism in the different cancer sites. Of note, the involvement of $X P A$ in cancer susceptibility may interact with other gene polymorphisms and with certain particular environmental exposures.

Due to the existence of several limitations, the results of the present meta-analysis should be interpreted with caution. First, the number of eligible published studies and the pooled sample size of independent studies were small in the overall and subgroup analyses, and it is possible that certain relevant unpublished studies were missed. Second, the effect of the confounding participants in gene-gene and gene-environment exposures and life habit interactions, such as the NER pathway, and tobacco and alcohol use, were not estimated in the present study due to data limitation. Therefore, to obtain a more precise analysis of XPA A23G polymorphism on OSCC risk, additional well-designed studies with larger sample sizes and diverse ethnicities are warranted. Regardless of these limitations, the present meta-analysis also has certain strengths. First, a systematic review of the association of the $X P A$ A23G polymorphism with the HNSCC risk is statistically more powerful than any independent study. Second, the quality of eligible studies included in the present meta-analysis was satisfactory without evidence of publication bias for the outcome (Table III).

In conclusion, the present meta-analysis provided a more precise estimation of the association between the XPA A23G polymorphism and HNSCC risk compared to independent studies. The results of the meta-analysis indicated that the $X P A$ A23G polymorphism may not be associated with overall HNSCC susceptibility but with oral carcinoma susceptibility, and it may be a risk factor for oral carcinoma; however, the study was a preliminary analysis and should be considered with caution. Further well-designed and large studies are required to clarify this association.

\section{Acknowledgements}

The present study was supported by National Natural Science Foundation of China (grant nos. 81400513, 81302358 and 81202132), the Science and Technology Commission of Shanghai (grant no. 14401931600), the Shanghai Education Commission Project of Young Teachers (grant no. ZZjdyx13104) and the Shanghai Natural Science Foundation (grant no. 13ZR1457100).

\section{References}

1. Siegel R, Naishadham D and Jemal A: Cancer statistics, 2012. CA Cancer J Clin 62: 10-29, 2012.

2. Marur S, D'Souza G, Westra WH and Forastiere AA: Hpv-associated head and neck cancer: a virus-related cancer epidemic. Lancet Oncol 11: 781-789, 2010.

3. Marron M, Boffetta P, Zhang ZF, et al: Cessation of alcohol drinking, tobacco smoking and the reversal of head and neck cancer risk. Int J Epidemiol 39: 182-196, 2010.

4. Leemans CR, Braakhuis BJ and Brakenhoff RH: The molecular biology of head and neck cancer. Nat Rev Cancer 11: 9-22, 2011.

5. Shell SM, Li Z, Shkriabai N, Kvaratskhelia M, et al: Checkpoint kinase ATR promotes nucleotide excision repair of UV-induced DNA damage via physical interaction with xeroderma pigmentosum group A. J Biol Chem 284: 24213-24222, 2009

6. Vaid M, Sharma SD and Katiyar SK: Proanthocyanidins inhibit photocarcinogenesis through enhancement of DNA repair and xeroderma pigmentosum group A-dependent mechanism. Cancer Prev Res (Phila) 3: 1621-1629, 2010.

7. Krasikova YS, Rechkunova NI, Maltseva EA, Petruseva IO and Lavrik OI: Localization of xeroderma pigmentosum group A protein and replication protein A on damaged DNA in nucleotide excision repair. Nucleic Acids Res 38: 8083-8094, 2010.

8. Liu J, Zhang Z, Cao XL, et al: XPA A23G polymorphism and susceptibility to cancer: a meta-analysis. Mol Biol Rep 39: 6791-6799, 2012.

9. Ding D, Zhang Y, Yu H, et al: Genetic variation of XPA gene and risk of cancer: a systematic review and pooled analysis. Int $\mathrm{J}$ Cancer 131: 488-496, 2012.

10. Lou Y, Li R, Zhang Y, et al: XPA gene rs1800975 single nucleotide polymorphism and lung cancer risk: a meta-analysis. Tumour Biol 35: 6607-6617, 2014.

11. Sugimura T, Kumimoto H, Tohnai I, et al: Gene-environment interaction involved in oral carcinogenesis: molecular epidemiological study for metabolic and DNA repair gene polymorphisms. J Oral Pathol Med 35: 11-18, 2006.

12. Hall J, Hashibe M, Boffetta P, et al: The association of sequence variants in DNA repair and cell cycle genes with cancers of the upper aerodigestive tract. Carcinogenesis 28: 665-671, 2006.

13. An J, Liu Z, Hu Z, et al: Potentially functional single nucleotide polymorphisms in the core nucleotide excision repair genes and risk of squamous cell carcinoma of the head and neck. Cancer Epidemiol Biomarkers Prev 16: 1633-1638, 2007.

14. Bau DT, Tsai MH, Huang CY, et al: Relationship between polymorphisms of nucleotide excision repair genes and oral cancer risk in Taiwan: evidence for modification of smoking habit. Chin J Physiol 50: 294-300, 2007.

15. Abbasi R, Ramroth H, Becher H, Dietz A, Schmezer P and Popanda O: Laryngeal cancer risk associated with smoking and alcohol consumption is modified by genetic polymorphisms in ERCC5, ERCC6 and RAD23B but not by polymorphisms in five other nucleotide excision repair genes. Int J Cancer 125: 1431-1439, 2009.

16. Jelonek K, Gdowicz-Klosok A, Pietrowska M, et al: Association between single-nucleotide polymorphisms of selected genes involved in the response to DNA damage and risk of colon, head and neck and breast cancers in a Polish population. J Appl Genet 51: 343-352, 2010. 
17. Lu B, Li J, Gao Q, Yu W, Yang Q and Li X: Laryngeal cancer risk and common single nucleotide polymorphisms in nucleotide excision repair pathway genes ERCC1, ERCC2, ERCC3, ERCC4, ERCC5 and XPA. Gene 542: 64-68, 2014.

18. Li X, Xu J, Yang X, et al: Association of single nucleotide polymorphisms of nucleotide excision repair genes with laryngeal cancer risk and interaction with cigarette smoking and alcohol drinking. Tumour Biol 35: 4659-4665, 2014.

19. Zhang L, Wang Y, Qiu Z, Luo J, Zhou Z and Shu W: The XRCC1 Arg194Trp polymorphism is not a risk factor for glioma: A meta-analysis involving 1,440 cases and 2,562 controls. Exp Ther Med 4: 1057-1062, 2012.

20. Zhuo XL, Ling JJ, Zhao HY, Zhou Y, Song YF and Tan YH Lack of association of the cyclin D1 G870A variation with oral carcinoma risk: Evidence from 2,404 subjects. Exp Ther Med 4: 748-754, 2012
21. Schena M, Guarrera S, Buffoni L, et al: DNA repair gene expression level in peripheral blood and tumour tissue from non-small cell lung cancer and head and neck squamous cell cancer patients. DNA Repair (Amst) 11: 374-380, 2012.

22. Azad AK, Bairati I, Samson E, et al: Validation of genetic sequence variants as prognostic factors in early-stage head and neck squamous cell cancer survival. Clin Cancer Res 18: 196-206, 2012

23. Song X, Sturgis EM, Jin L, Wang Z, Wei Q and Li G: Variants in nucleotide excision repair core genes and susceptibility to recurrence of squamous cell carcinoma of the oropharynx. Int J Cancer 133: 695-704, 2013. 